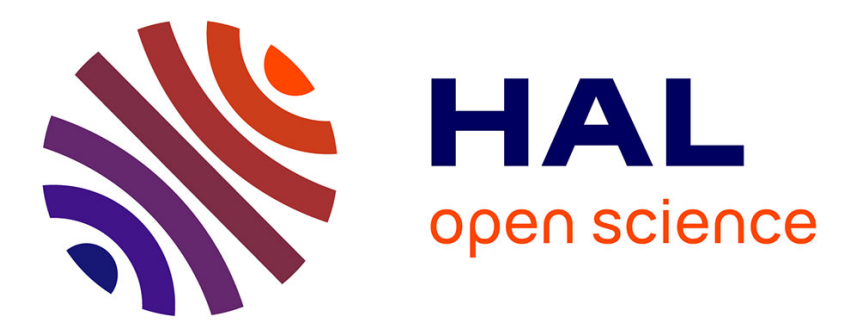

\title{
A micromechanical explanation of the mean stress effect in high cycle fatigue
}

\author{
Vincent Monchiet, Eric Charkaluk, Djimedo Kondo
}

\section{To cite this version:}

Vincent Monchiet, Eric Charkaluk, Djimedo Kondo. A micromechanical explanation of the mean stress effect in high cycle fatigue. Mechanics Research Communications, 2008, 35 (6), pp.383-391. 10.1016/j.mechrescom.2008.03.004 . hal-00333028

\section{HAL Id: hal-00333028 \\ https://hal.science/hal-00333028}

Submitted on 27 Jul 2021

HAL is a multi-disciplinary open access archive for the deposit and dissemination of scientific research documents, whether they are published or not. The documents may come from teaching and research institutions in France or abroad, or from public or private research centers.
L'archive ouverte pluridisciplinaire HAL, est destinée au dépôt et à la diffusion de documents scientifiques de niveau recherche, publiés ou non, émanant des établissements d'enseignement et de recherche français ou étrangers, des laboratoires publics ou privés. 


\title{
A micromechanical explanation of the mean stress effect in high cycle fatigue
}

\author{
V. Monchiet, E. Charkaluk*, D. Kondo \\ Laboratoire de Mécanique de Lille - UMR CNRS 8107, Université de Sciences et Technologies Lille, Cité Scientifique, Bd. Paul Langevin, \\ 59655 Villeneuve d'Ascq Cedex, France
}

\begin{abstract}
A micro-macro approach of multiaxial fatigue in unlimited endurance is presented in this study, as an extension of a previous model recently proposed by the authors [Monchiet, V., Charkaluk, E., Kondo, D., 2006. A plasticity-damage based micromechanical modelling in high cycle fatigue. C.R. Mécanique 334 (2), 129-136]. It allows to take into account coupling between polycrystalline plasticity and damage mechanisms which occur at the scale of persistent slip bands (PSB) during cyclic deformation. The plasticity-damage coupled model is obtained by adapting the Gurson [Gurson, A.L., 1977. Continuum theory of ductile rupture by void nucleation and growth: part I - yield criteria and flow rules for porous ductile media. J. Eng. Mater. Technol. 99, 2-15] limit analysis to polycrystalline materials to take into account microvoids growth along PSBs. The macroscopic fatigue criterion corresponds to microcracks nucleation at the PSB-matrix interface. It is shown that this criterion accounts for the effect of the mean stress and of the hydrostatic pressure in high cycle fatigue. Such features of HCF are related to the damage micro-mechanisms. Finally, some illustrations concerning the particular case of cyclic affine loadings are presented and comparisons of the predictions of the fatigue criterion with experimental data show the relevance of this new approach.
\end{abstract}

\section{Introduction}

It is now generally admitted that fatigue failure of metallic components is the result of complex microscopic phenomena which occur at the grain scale under cyclic loadings. In particular, a major role is assigned to the microplasticity due to the dislocations motion and to the damage by microcracks growth (Dang Van, 1999; Essmann and Mughrabi, 1979). The objective of this study is to propose a high cycle fatigue (HCF) criterion founded on the physical microscopic mechanisms leading to damage and cracks nucleation.

In HCF context, many multiaxial fatigue criteria already exist. Among the first attempts dealing with purely phenomenological approach, mention can be made of Sines (1959) or Crossland (1956) macroscopic criteria. The first real studies based on microscopic observations for the expression of a macroscopic criterion are those of Orowan (1939) then of Dang Van (1973), who benefited of a lot of experimental results and observations obtained between the 30s and the 60s. This multiscale approach was thereafter enriched and formalized by Papadopoulos (1987). The theoretical framework proposed by Dang Van and Papadopoulos is based on the modeling of the plasticity at the grain scale and on the use of elastic shakedown theorems in order to establish a crack nucleation criterion. It is worth noticing that in this type of modeling, despite the clear physical reference to damage phenomena, no explicit introduction of the local damage mechanisms is done.

\footnotetext{
* Corresponding author.

E-mail address: eric.charkaluk@univ-lillel.fr (E. Charkaluk).
} 
In a recent work (Monchiet et al., 2006), we have proposed a multiscale approach of HCF based on the Dang Van and Papadopoulos theoretical background and on the consideration of plasticity and damage mechanisms at the scale of the grains. The damage mechanisms, which occur along highly deformed slip bands, named persistent slip bands (PSB) in the fatigue case, are assumed of two types: on the one hand, microvoids nucleation by accumulation of point defects of vacancies type, generated by annihilation of dislocations (Essmann and Mughrabi, 1979), and, on the other hand, the growth of these microvoids under the combined effect of the slip-like plastic activity and of the pressure. In the case of von Mises matrix material, this defect growth is generally described in the ductile damage framework, classically defined under monotonic loadings (Rice and Tracey, 1969; Gurson, 1977). The model proposed in Monchiet et al. (2006) was based on a simple adaptation of the Rice and Tracey's voids growth approach (Rice and Tracey, 1969) and led to the expression of a fatigue criterion exhibiting the crucial role of the hydrostatic pressure in high cycle fatigue. The essential feature of this first model is that damage and plasticity are uncoupled, which facilitates the numerical implementation of the criterion. However, it is convenient to note that with such a model the role of an alternated part of the hydrostatic pressure is not satisfactorily represented in view of available data.

In order to properly describe the growth of the microcavities along PSBs as well as the role of the alternated part of the hydrostatic pressure, it is proposed in this paper to adapt the limit analysis of Gurson (1977) which has the interest of coupling plasticity and damage in the constitutive law. An hollow sphere representing an elementary cell of the monocrystal containing microcavities is therefore considered and serves as the geometrical model for the analysis of a porous material governed by an equivalent von Mises criterion corresponding to the monocrystal.

Since an essential step in the treatment of fatigue type cyclic loadings is the consideration of plastic hardening, it is proposed to incorporate the hardening effects by following the previous study of Leblond et al. (1995), extended here to crystalline plasticity. The adopted crack initiation criterion corresponds to a critical value of damage at the PSB's scale. In order to upscale this criterion to the macroscopic scale, a micro-macro transition based on the Kröner's self-consistent scheme (Kröner, 1961) is used. This micromechanical reasoning leads to a closed form expression of the fatigue criterion in the case of affine loading paths. In order to evaluate the relevance of this approach, comparisons with available experimental data are proposed in a last part of this article.

\section{Basic principle of the modeling}

\subsection{Plastic behavior at the grain scale}

In this section, plastic micromechanisms at the grain scale, corresponding to an activation of slip systems are described. In the case of HCF, for FCC structures, a simple slip system assumption is commonly adopted (Dang Van, 1973; Papadopoulos, 1987). This assumption is mainly justified for low stress amplitudes, below the macroscopic yield stress. Let $\sigma$ and $\varepsilon$ be, respectively, the stress and strain tensors at the local scale, i.e. at the grain scale. An additive partition of the total strain $\varepsilon$ in an elastic part $\varepsilon^{e}$ and a plastic part $\varepsilon^{p}$ is adopted: $\varepsilon=\varepsilon^{e}+\varepsilon^{p}$. The plastic slip, on the activated slip system, is defined by $\gamma^{p}=2 \varepsilon^{p}: \Delta$, where $\Delta$ is the orientation tensor, defined by: $\Delta=\underline{n} \stackrel{s}{\otimes} \underline{m}=\frac{1}{2}(\underline{n} \otimes \underline{m}+\underline{m} \otimes \underline{n})$. The vector $\underline{n}$ is the normal to the activated slip plane while $\underline{m}$ is the slip direction. The plastic behavior of this monocrystal gives relation between the shear stress $\tau=\boldsymbol{\sigma}: \Delta$ and the plastic slip strain $\gamma^{p}$; as classically, a Schmid's law is considered

$$
|\tau-X|-\tau_{0}-R=0
$$

$X$ and $R$ are, respectively, the kinematic and isotropic hardening variables. A linear law is adopted for both hardenings: $X=c \gamma^{p}$ and $R=R_{0} p$. The positive scalars $c$ and $R_{0}$ are the hardening moduli and $p=\int_{0}^{t}\left|\gamma^{j p}\right| \mathrm{d} t^{\prime}$ is the cumulated plastic slip.

\subsection{Damage modeling}

\subsubsection{Voids nucleation and growth mechanisms}

According to previous assumptions, the proposed multiscale modeling is based on irreversible mechanisms which deteriorate definitively the microstructure. In the case of FCC crystals and cyclic loadings, the plastic strain localization induces dislocations annihilation mechanism which is at the origin of point defects accumulation of vacancies type along PSBs. Dislocations annihilation is not an irreversible mechanism: point defects could be mutually annihilated. A phenomenological model for the production of defects by dislocations annihilation was previously proposed by Essmann and Mughrabi (1979). For low values of $p$, which correspond to the HCF situation (Monchiet et al., 2006), the porosity induced by this mechanism, denoted $\eta_{a}$, is given by

$$
\eta_{a}(p)=A_{0}\left[k_{a} p-1+\exp \left(-k_{a} p\right)\right]
$$

where $A_{0}$ and $k_{a}$ are two parameters. This annihilation mechanism depends only on the plastic slip; as a consequence, it is not able to predict the important role of the hydrostatic pressure observed in fatigue. A possible way to overcome such limitation is to consider a second damage mechanism in addition to the annihilation's one and which may depend on the hydrostatic pressure. As microcavities nucleation is induced by vacancies production, they are supposed to grow under the combined effect of the plastic strains of the matrix and of the pressure, as underlined by Rice and Tracey (1969) under monotonic loadings. Therefore, the porosity $\eta_{g}$, corresponding to the microcavities growth, is introduced. The total porosity $\eta$ is 
then the sum of the porosities $\eta=\eta_{a}+\eta_{g}$. The growth of the cavities induces plastic volume variations which result in a plastic deformation by cavities expansion; this implies that the plastic strain at the grain scale is partitioned as follows:

$$
\varepsilon^{p}=\gamma^{p} \Delta+\varepsilon_{h}^{p} 1
$$

where the hydrostatic part of the plastic strain $\varepsilon_{h}^{p}$ due to voids growth can be related to $\eta_{g}$ by considering mass balance, in the case of limited porosities

$$
\dot{\eta}_{g}=(1-\eta) 3 d_{h} \dot{\varepsilon}_{h}^{p} \simeq 3 \dot{\varepsilon}_{h}^{p}
$$

and in the case where the elastic strain of the matrix has been neglected. Moreover, one supposes that a no crack initiation criterion is obtained by considering that no crack can be observed at the grain size; this can be related to a critical value of the total porosity, denoted $\eta_{c}$. The fatigue criterion reads then

$$
\eta=\eta_{a}(p)+\eta_{g}\left(\varepsilon_{h}^{p}\right)=\eta_{c}
$$

It is interesting to note that this criterion depends on $p$ and on $\varepsilon_{h}^{p}$ and generalize the approaches considering a critical value of the cumulated plastic strain $p$ (Papadopoulos criterion for example (Papadopoulos, 1996)). As exhibited by Eq. (5), one can underline that the general basis of this approach consists in the definition of a defect nucleation law, corresponding here to $\eta_{a}$, associated to a damage evolution, conducting here to $\eta_{g}$. Then, in many cases, PSBs are not systematically observed at the microstructure scale. Particularly, in a lot of engineering cases, it is well known that initial defects, as inclusions, precipitates, are the origin of the fatigue crack initiation. The proposed criterion corresponding to Eq. (5) is, however, still acceptable, but the definition of $\eta_{a}$ has to be adapted, for example by taking into account an initial porosity due to the process. Then, the initial defect's evolution is still governed by the combined effect of local plastic strains of the matrix and of hydrostatic pressure.

Now, as previously mentioned, the first step of modeling proposed in Monchiet et al. (2006) provided a Rice and Tracey type (Rice and Tracey, 1969) evolution law for $\varepsilon_{h}^{p}$. The next step consists now in the definition of the constitutive law coupling plasticity and damage in order to link the strains defined at the grain scale and the associated stresses.

\subsubsection{Coupled plasticity-damage at the grain scale}

We aim now at considering microvoids growth coupled with the plasticity in a PSB. To this end, we will adapt the limit analysis framework derived by Gurson (1977). Due to the difficulties introduced by the anisotropic character of the crystalline plasticity, a first step of the modeling consists in the replacement of the monocrystal obeying the Schmid's law by an equivalent material governed by the von Mises criterion. An elementary cell consisting in an hollow sphere of radius $b$ containing a spherical cavity of radius $a$ is then considered (see Fig. 1); the porosity is $\eta=(a / b)^{3}$. The hollow sphere is submitted to a uniform strain rate on its external boundary: $\underline{v}(r=b)=\mathbf{D} \cdot \underline{x} ; \underline{v}$ is the velocity field in the matrix. D contains a simple slip whose orientation is given by $\Delta$ and an hydrostatic strain rate, i.e.

$$
\mathbf{D}=\gamma^{p} \boldsymbol{\Delta}+D_{h} 1
$$

Following then the limit analysis approach of Gurson for the above particular boundary conditions (6), an explicit expression of the yield condition is obtained (for more details, see Monchiet, 2006)

$$
\mathscr{F}=\frac{(\boldsymbol{\sigma}: \boldsymbol{\Delta})^{2}}{\tau_{0}^{2}}+2 \eta \cosh \left\{\frac{\sqrt{3}}{2} \frac{\sigma_{h}}{\tau_{0}}\right\}-1-\eta^{2}
$$

As for the Gurson's criterion, Eq. (7) indicates that the porosity $\eta$ affects the macroscopic plasticity of the matrix. Moreover, it shows a dependence on the hydrostatic pressure. In the case of an equally null porosity $(\eta=0)$, the plastic criterion reduces as it should be to the Schmid's law, in perfect plasticity, for the activated slip system.

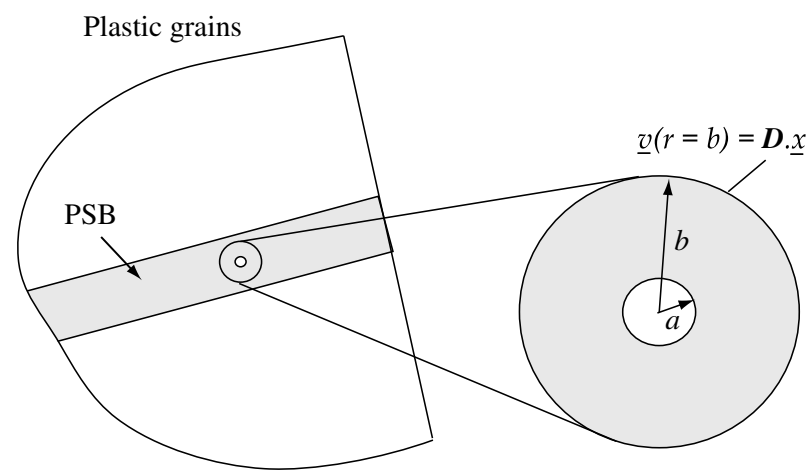

Fig. 1. Elementary cell. 
We need now to extend (7), established in the case of the perfect plasticity, to the context of plasticity with hardening. Indeed, despite the fact that perfect plasticity generally allows first approximations of mechanical response, it is not adapted to cyclic loadings. As shown by Papadopoulos (1987), hardening laws has to be considered as monocrystals submitted to cyclic loadings exhibit isotropic and kinematic hardening characters. Such an extension of the original criterion of Gurson was previously proposed by Leblond et al. (1995), by using a micromechanical reasoning. Adapting then this extension, it is assumed here that the expression of the macroscopic yield function with isotropic and kinematic hardening has the following form:

$$
\mathscr{F}=\left(\frac{\mathbf{B}: \Delta}{\tau_{d}}\right)^{2}+2 \eta \cosh \left\{\frac{\sqrt{3}}{2} \frac{B_{h}}{\tau_{h}}\right\}-1-\eta^{2}
$$

where $\mathbf{B}=\boldsymbol{\sigma}-\mathbf{X}, \mathbf{X}$ is the kinematic hardening variable, defining the center of the elastic domain. $\mathbf{X}$ is partitioned in an hydrostatic part $X_{h}\left(X_{h}=\operatorname{tr}(\mathbf{X}) / 3\right)$ and a deviatoric part $X_{d}$, defined by $X_{d}=2 \mathbf{X}: \Delta$. To take into account the isotropic part of the hardening, the yield stress $\tau_{0}$ is replaced by two parameters $\tau_{d}$ and $\tau_{h}$, defined by: $\tau_{d}=\tau_{0}+R_{d}$ et $\tau_{h}=\tau_{0}+R_{h}$. The quantities $R_{d}$ and $R_{h}$ are the two variables of the isotropic hardening. The problem to solve consists then in the determination of the parameters $X_{d}, X_{h}, R_{d}$ and $R_{h}$.

The expressions of the hardening parameters are determined by considering particular loading paths for which an exact solution of the limit analysis problem can be identified. This method, developed in Monchiet (2006) in the context of crystalline plasticity, leads to the following expressions for the hardening parameters involved in (8):

$$
R_{d}=R_{0} p ; \quad X_{d}=(1-\eta) c \gamma^{p} ; \quad X_{h}=\frac{2 c}{\sqrt{3}} \alpha ; \quad R_{h}=-\frac{R_{0}}{\ln (\eta)} \alpha_{c u m}
$$

in which has been introduced

$$
\alpha=\frac{2}{\sqrt{3}}\left[\operatorname{dilog}\left(\frac{\eta_{a}}{\eta}\right)-\eta_{g}\right]
$$

and $\alpha_{\text {cum }}=\int_{0}^{t}|\dot{\alpha}| \mathrm{d} t^{\prime}$ which is the cumulated value of the variable $\alpha$.

\subsubsection{Evolution laws}

The evolution laws of the variables $p$ and $\varepsilon_{h}^{p}$, which appear explicitly in the expression of the fatigue criterion (5), are obtained via the normality rule ${ }^{1}$ associated to $\mathscr{F}$ Eq. (8)

$$
\dot{\boldsymbol{\varepsilon}}^{p}=\dot{\lambda} \frac{\partial \mathscr{F}}{\partial \boldsymbol{\sigma}} \Rightarrow\left\{\begin{array}{l}
\dot{p}=2 \dot{\lambda} \frac{|\mathbf{B}: \Delta|}{\tau_{d}^{2}} \\
\dot{\varepsilon}_{h}^{p}=\dot{\lambda} \frac{\eta}{\sqrt{3} \tau_{h}} \sinh \left\{\frac{\sqrt{3}}{2} \frac{B_{h}}{\tau_{h}}\right\}
\end{array}\right.
$$

$\dot{\lambda}$ corresponds to the plastic multiplier. Eq. (11) of $p$ and $\varepsilon_{h}^{p}$ depend on the local stress tensor, i.e. on $\tau$ and $\sigma_{h}$.

We need now to formulate the fatigue criterion at the macroscopic scale, i.e. as a function of the macroscopic stress tensor, $\boldsymbol{\Sigma}$. For this purpose, a micro-macro transition is due.

\section{Micro-macro transition}

In the context of HCF, one must remember that plasticity remains confined in a few grains, favorably oriented with respect to the loading axes, i.e. on which plastic slip is more easier. The macroscopic behavior is not affected by this plasticity and remains elastic. Let us denote by $\boldsymbol{\Sigma}$ the macroscopic stress tensor and by $\mathbf{E}$ the macroscopic strain tensor. These tensors are related by the Hooke's law: $\mathbf{\Sigma}=\mathbb{C}: \mathbf{E}$, where $\mathbb{C}$ is the stiffness tensor of the aggregate, which is considered isotropic for sake of simplicity. The elastic behavior of the inclusion (i.e. the unfavorably oriented grain) is defined by $\sigma=\mathbb{C}^{I}: \boldsymbol{\varepsilon}^{e}$. The general case corresponds to $\mathbb{C}^{I} \neq \mathbb{C}$, as the elastic behavior of the grain is classically characterized by a cubic elastic law; nevertheless, for sake of simplicity, this anisotropy is neglected and $\mathbb{C}^{I}=\mathbb{C}^{2}$. It is emphasized that the context of metals HCF corresponds to the beginning of monotonic plasticity (confined plasticity) for which the Eshelby-Kröner's approach is completely justified (see (Bornert et al., 2006)). Then, applying the Kröner's self-consistent scheme (Kröner, 1961), the interaction law is given by

$$
\boldsymbol{\sigma}=\boldsymbol{\Sigma}-\mathbb{C}^{*}: \varepsilon^{p}
$$

where $\mathbb{C}^{*}=\mathbb{C}:(\mathbb{Q}-\mathbb{P}: \mathbb{C}), \mathbb{P}$ is the Hill's fourth order tensor which depends on the elastic properties of the matrix and of the inclusion geometry. The expressions of the hydrostatic pressure, $\sigma_{h}$, and of the shear stress, $\tau$, involved in (7) and (11) and required for the determination of the macroscopic criterion (5) are readily obtained as

\footnotetext{
1 As classically, it is demonstrated that this is the consequence of the normality rule at the local scale.

2 From the theoretical point of view, taking into account the cubic symmetry of the grain does not introduce major difficulties. This has been done in Monchiet (2006).
} 


$$
\tau=\boldsymbol{\Sigma}: \Delta-\mu^{*} \gamma ; \quad \sigma_{h}=\Sigma_{h}-3 k^{*} \varepsilon_{h}^{p}
$$

with: $k^{*}=\frac{2 k}{3} \frac{1-2 v}{1-v}$ and $\mu^{*}=\frac{\mu}{15} \frac{7-5 v}{1-v}$. The upscaling of the local constitutive law, defined by (8) and (11), at the macroscopic scale is obtained from (13) and leads to an apparent hardening, denoted $X_{d}^{*}=X_{d}+\mu^{*} \gamma$ and $X_{h}^{*}=X_{h}+3 k^{*} \varepsilon_{h}^{p}$. The yield function (8) can then be written

$$
\mathscr{F}=\left(\frac{\boldsymbol{\Sigma}: \Delta-X_{d}^{*}}{\tau_{h}}\right)^{2}+2 \eta \cosh \left\{\frac{\sqrt{3}}{2} \frac{\Sigma_{h}-X_{h}^{*}}{\tau_{h}}\right\}-1-\eta^{2}
$$

and the plastic strain evolution laws

$$
\begin{aligned}
& \dot{\gamma}_{\text {cum }}=2 \dot{\lambda} \frac{\left|\boldsymbol{\Sigma}: \Delta-X_{d}^{*}\right|}{\tau_{d}^{2}} \\
& \dot{\varepsilon}_{h}^{p}=\dot{\lambda} \frac{\eta}{\sqrt{3} \tau_{h}} \sinh \left\{\frac{\sqrt{3}}{2} \frac{\Sigma_{h}-X_{h}^{*}}{\tau_{h}}\right\}
\end{aligned}
$$

It must be emphasized that the numerical integration of such a model at the macroscopic scale is quite not modified by this apparent hardening. More generally, the macroscopic stress is a function of time, describing a closed curve which corresponds to the cyclic loading path, in the stress space. The determination of the fatigue criterion is possible by means of the integration on the loading path of the constitutive law defined by (14). The no crack nucleation condition defined by (5) has then to be verified. As an example, an application of this criterion is now proposed in the case of affine macroscopic loading paths.

\section{The case of affine loading paths}

\subsection{Application of the model to affine loading paths}

When the cyclic loading path is affine, the macroscopic stress tensor has the following form: $\boldsymbol{\Sigma}(t)=\Sigma_{a} \sin (\omega t)+\Sigma_{m}$, where $\Sigma_{a}$ and $\Sigma_{m}$ are, respectively, the alternated and mean parts of the macroscopic stress tensor whose shear components are denoted $T_{a}=\Sigma_{a}: \Delta$ and $T_{m}=\Sigma_{m}: \Delta$, and the hydrostatic parts, $\Sigma_{h, a}$ and $\Sigma_{h, m}$. It is well known that, in this particular case, the multiaxial loading path reduces as a straight line in the stress space.

In case of macroscopic affine loading paths, the asymptotic behavior of plastic structures is characterized by elastic shakedown, plastic shakedown or ratcheting. In both last cases, the failure is the result of the accumulation of plastic strains and damage along PSBs. A necessary condition of no crack initiation is to ensure elastic shakedown at the local scale. This first subsection presents numerical results obtained with the coupled plasticity-damage model presented in the previous sections, in the case of affine loadings. For the different applications, the assumed parameters of the model are given in Table 1. The order of magnitude of these parameters is deduced from the literature on monocrystals cyclic behavior (such as (Winter, 1974; Essmann and Mughrabi, 1979), a general review of this literature can be founded in Suresh (1998)) and these values simply give general evolutions of the model. As it will be underlined in the next part, for a given material, these parameters are not determined individually but globally, as a combination, from macroscopic fatigue limit.

The plastic criterion at the shakedown state in the macroscopic stress space $\left(\Sigma: \Delta, \Sigma_{h}\right)$ is represented in Fig. 2 for the following particular values: $T_{a}=200 \mathrm{MPa}, T_{m}=0, \Sigma_{h, a}=600 \mathrm{MPa}$ and $\Sigma_{h, m}=200 \mathrm{MPa}$. As only the shear component on the slip plane and the hydrostatic pressure are considered, the macroscopic stress space can be reduced to this $2 \mathrm{D}$ representation. It can be noted that the obtained surface is symmetric, centered on the macroscopic stress path (represented by the segment $A B$ ), i.e. around $T_{m}=0$ and $\sigma_{h, m}=200 \mathrm{MPa}$. This feature has been verified more generally: in fact, at the local scale of the grain, at the shakedown state, the stress cycle is defined by $\sigma=\Sigma_{a} \sin (\omega t)$; the residual stress tensor balances with the macroscopic mean stress tensor, i.e. $\boldsymbol{\rho}=\boldsymbol{\Sigma}_{m}$. The three following relations are deduced from these observations:

$$
\begin{aligned}
& T_{m}=X_{d}^{*}=\left(\left(1-\eta_{c}\right) c+\mu^{*}\right) \gamma^{p} \\
& \Sigma_{h, m}=X_{h}^{*} \\
& \frac{T_{a}^{2}}{\tau_{d}^{2}}+2 \eta_{c} \cosh \left\{\frac{\sqrt{3}}{2} \frac{\Sigma_{h, a}}{\tau_{h}}\right\}-1-\eta_{c}^{2}=0
\end{aligned}
$$

Table 1

Assumed model's parameter

\begin{tabular}{llllll}
\hline$\tau_{0}$ & $R_{0}$ & $c$ & $A_{0}$ & $k$ & $k$ \\
\hline $60 \mathrm{MPa}$ & $20 \mathrm{MPa}$ & $1000 \mathrm{MPa}$ & 0.0008 & 2 & $200 \mathrm{GPa}$ \\
\hline
\end{tabular}

The order of magnitude of these parameters is deduced from the literature on monocrystals cyclic behavior (Winter, 1974; Essmann and Mughrabi, 1979; Suresh, 1998). 


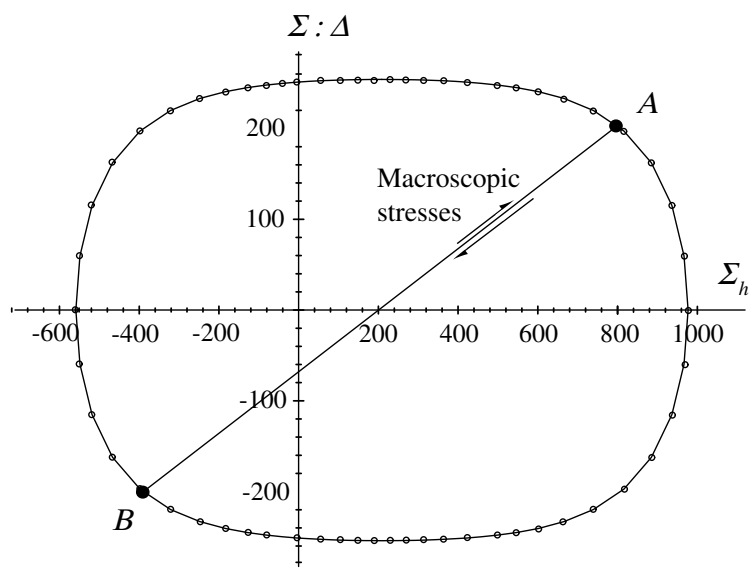

Fig. 2. Loading surface in the plane $\left(\Sigma: \Delta, \Sigma_{h}\right)$, obtained by numerical simulation using the equations of the coupled plasticity-damage model proposed in the previous parts associated to the parameters coming from Table 1.

These new results, obtained in the case of a plasticity depending on the hydrostatic pressure, extend those of Papadopoulos (1987) in classical plasticity. In Monchiet et al. (2006), the role of the mean stress, and, in particular, of the mean part of the hydrostatic pressure, on the fatigue criterion was clearly demonstrated in the case of an uncoupling between plasticity and damage. This effect of the mean stress is recovered here, as shown in Fig. 3. In fact, the amount of total porosity, which represents fatigue damage in the present model, obtained at the shakedown state, depends clearly on the shear stress amplitude and also on the mean part of the hydrostatic pressure. One of the important contribution of the present study is to provide a physical explanation of the crucial role of the alternated part of the hydrostatic pressure. In Fig. 4, the cumulated plastic slip $p$ is represented as a function of the hydrostatic pressure amplitude $\Sigma_{h, a}$, for a shear amplitude of $T_{a}=200 \mathrm{MPa}$. The variation of $p$ with this component of the hydrostatic pressure illustrates the dependence of the plastic behavior on $\Sigma_{h}$ and then, the importance of the plasticity-damage coupling. Indeed, the dotted line in Fig. 4 corresponds to the uncoupled model presented in Monchiet et al. (2006) and which shows the independence of $p$ with respects to $\Sigma_{h, a}$ in this case. The dependence of the porosity value $\eta$ with the hydrostatic pressure amplitude, $\Sigma_{h, a}$, is besides observed in Fig. 5, where the variations of the porosity $\eta$ are presented as a function of $\Sigma_{h, a}$ for different values of the shear amplitudes $T_{a}$. Once again, this dependence illustrates the coupling between loading surface, damage and hydrostatic pressure and the particularity of the proposed HCF model.

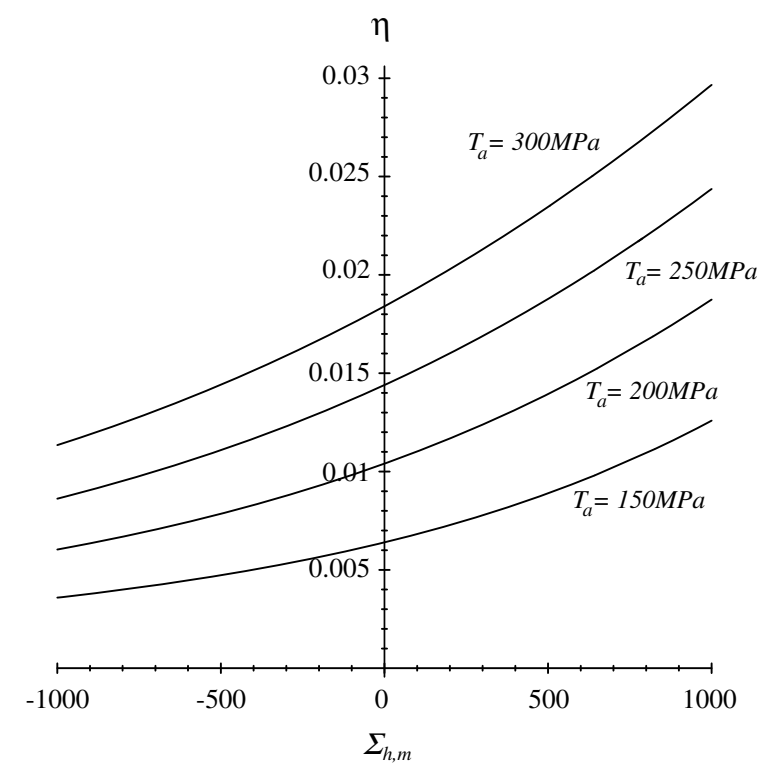

Fig. 3. Variation of the porosity $\eta$ with the mean hydrostatic pressure $\Sigma_{h, m}$ for different values of the shear amplitude $T_{a}$. 


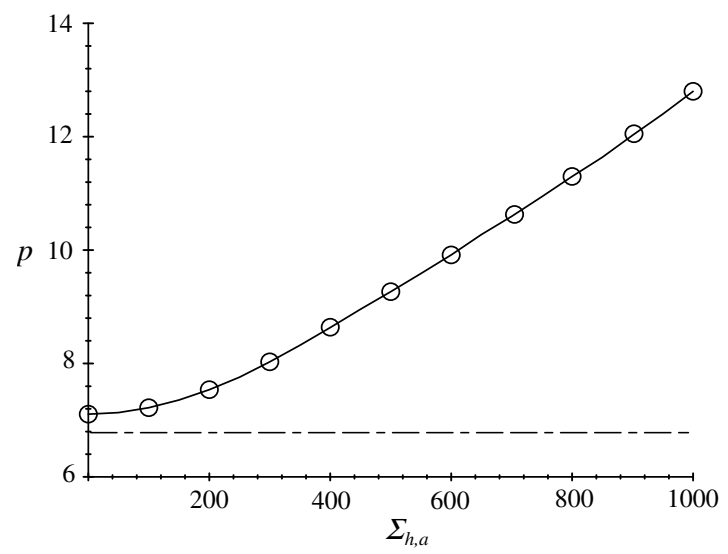

Fig. 4. $p$ at the shakedown state as a function of $\Sigma_{h, a}$, for $T_{a}=200 \mathrm{MPa}$. The dotted line corresponds to the uncoupled model presented in Monchiet et al. (2006) and the plain line to the present coupled model.

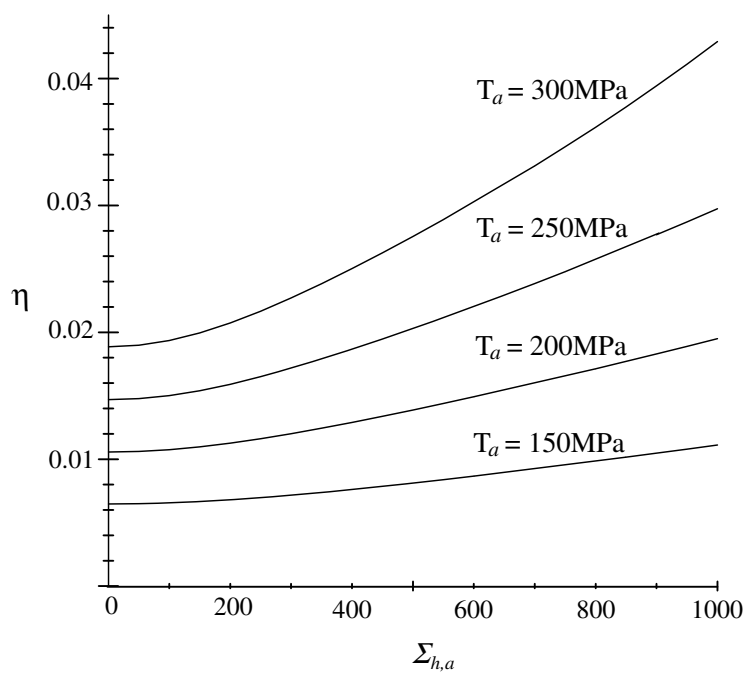

Fig. 5. Variation of the porosity $\eta$ with the amplitude of the hydrostatic pressure $\Sigma_{h, a}$ for different values of the shear amplitude $T_{a}$.

\subsection{Illustration on macroscopic affine loadings}

In the previous section, the symmetrization of the microscopic loading cycle at the shakedown state has been verified in the case of macroscopic affine loading paths. This observation leads to three relations given by (16). One can then first remark that the fatigue criterion is only a function of $p$ and $\varepsilon_{h}^{p}$ but not of the plastic slip $\gamma^{p}$ at the shakedown state. Taking into account the first relation in (16), this implies that the criterion is independent of the macroscopic mean shear stress which is in accordance with many experimental results already reported in literature (Sines, 1959). From the two last relations of (16), the local variables $p$ and $\varepsilon_{h}^{p}$ can be deduced from the macroscopic variables $T_{a}, \Sigma_{h, a}, \Sigma_{h, m}$. Therefore, in a general way, it can be stated that the proposed criterion is based on the same variables as the Dang Van's criterion (Dang Van, 1999). However, in the present approach, the dependence on the alternated and mean components of the hydrostatic pressure, $\Sigma_{h, a}$ et $\Sigma_{h, m}$, results clearly from the explicit incorporation of the damage mechanisms along the PSBs in the multiscale modeling. As a first illustration, results of Ros (1950) corresponding to reversed torsion tests with mean tension on En25T steel are considered. The Fig. 6 shows a good agreement between the different models and experimental results and illustrates the influence of the mean stress, represented here by the mean part of the hydrostatic pressure. It is interesting to note that in this case, the uncoupled model proposed in Monchiet et al. (2006) corresponds exactly to the Dang Van's criterion, as demonstrated in Monchiet, 2006. In this illustration, as well as in the following, the combinations of the parameters of the model are globally identified in these more simple loading cases, directly from two experimental points. In order to examine the particular role of the alternated part of the hydrostatic pressure, the well known experimental results of Gough and Pollard (1935) for reversed bending-torsion tests are considered. In Fig. 7, they are represented in the plane $\left(\Sigma_{12, a} ; \Sigma_{11, a}\right)$ corresponding, respectively, to the torsion and bending parts of the loading. As 


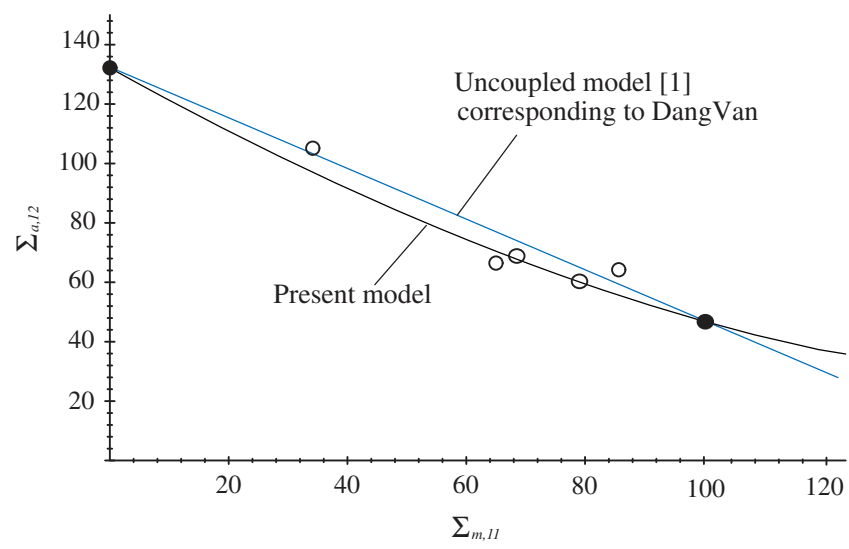

Fig. 6. Repeated torsion with mean tension on En25T steel: comparison of the present criterion with the uncoupled model (Monchiet et al., 2006), the Dang Van criterion and the experimental data of Ros (1950) (o). The experimental points used for the global identification of the model's parameters are in black.

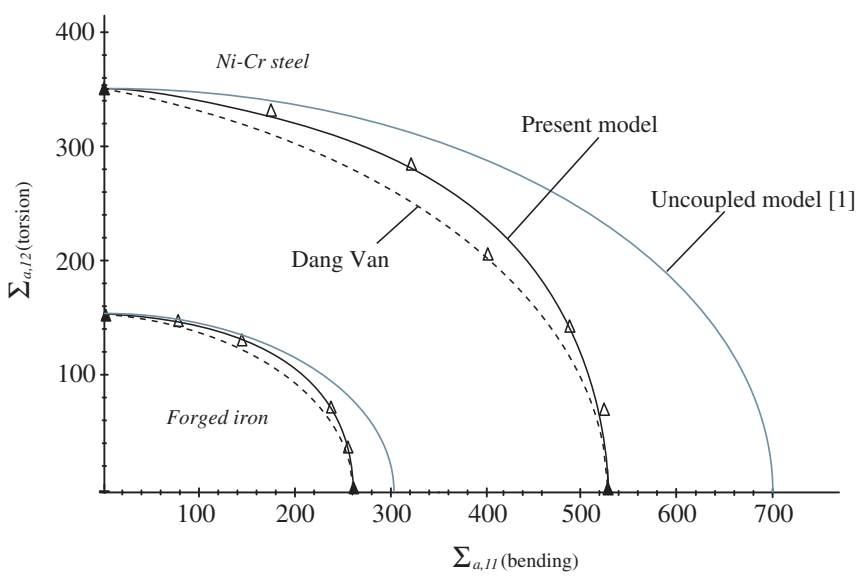

Fig. 7. Reversed bending-torsion on forged iron and Ni-Cr steel: comparison of the present criterion with the uncoupled model (Monchiet et al., 2006), the Dang Van criterion and the experimental data of Gough and Pollard $(1935)(\Delta)$. The experimental points used for the global identification of the model's parameters are in black.

emphasized in the introduction, the uncoupled approach proposed in Monchiet et al. (2006) does not take into account the alternated part of the hydrostatic pressure, which explains this gap between this model and the experimental data. The Dang Van criterion is in a relative accordance with the experimental data. One must recall in this particular case that a linear relation is postulated between the local shear stress and the maximum hydrostatic pressure which includes its alternated part. At last, the proposed fatigue criterion, directly deduced from a plasticity-damage based micromechanical approach, enables a more physical representation of the role of the alternated part of the hydrostatic pressure, as well as of its mean part. Compared to Dang Van's approach, for which elastic shakedown corresponds to the fatigue criterion, one of the principal interest of this micromechanical fatigue model is that fatigue criterion is defined as a critical damage value $\eta_{c}$. Then, damage evolution laws are defined in the second part of this paper, depending on the plastic strains evolution laws described through the Eq. (11). Therefore, this approach enables the treatment of any complex loadings, as variable amplitude ones. This particular case can not be analyzed with Dang Van's criterion without rough approximations and assumptions.

\section{Conclusion}

A multiscale approach of the determination of a High Cycle Fatigue criterion has been proposed. It allows to take into account, not only plasticity activity in some grains, but also damage due to microvoids growth along PSB-matrix interface. The proposed criterion is obtained by adapting the extension of Gurson analysis by Leblond et al., extended here to cyclic loadings and hardening crystalline plasticity. It allows to demonstrate a significant effect of the mean pressure as well as of its alternated part. This effect is clearly related to the considered damage mechanisms. It is believed that the proposed approach and the obtained results open new perspectives in fatigue, as they can be extended to the treatment of unconfined (macroscopic) plasticity and thermomechanical cyclic loadings which remain open engineering problems. 


\section{References}

Bornert, M., Bretheau, T., Gilormini, P., 2006. Homogenization in Mechanics of Materials. Iste Publishing Company.

Crossland, B., 1956. Effects of large hydrostatic pressures on tortional fatigue strength of an alloy steel. In: Proceedings of the International Conference on Fatigue of Metals. London Institution of Mechanical Engineers, p. 138

Dang Van, K., 1973. Sur la résistance à la fatigue des métaux. Sci. Tech. Armement 47 (3).

Dang Van, K., 1999. Introduction to fatigue analysis in mechanical design by the multiscale approach. In: High-Cycle Fatigue in the Context of Mechanical Design. CISM Courses and Lectures, 392. Springer-Verlag, pp. 1691-1710.

Essmann, U., Mughrabi, H., 1979. Annihilation of dislocations during tensile and cyclic deformation and limits of dislocation densities. Philos. Mag. A 40 (6), 731-756.

Gough, H.J., Pollard, H.V., 1935. The strength of metals under combined alternating stress. Proc. Inst. Mech. Eng. 131, 3-18.

Gurson, A.L., 1977. Continuum theory of ductile rupture by void nucleation and growth: part I - yield criteria and flow rules for porous ductile media. J. Eng. Mater. Technol 99, 2-15.

Kröner, E., 1961. Zur plastishen verformung des vielkristalls. Acta Metall. 9, 155-161.

Leblond, J.B., Perrin, G., Devaux, J., 1995. An improved Gurson-type model for hardenable ductile metals. Eur. J. Mech. A/Solids 14 (4), $499-527$.

Monchiet, V., Charkaluk, E., Kondo, D., 2006. A plasticity-damage based micromechanical modelling in high cycle fatigue. C.R. Mécanique 334 (2), $129-136$.

Monchiet, V., 2006. Contributions à la modélisation micromécanique de l'endommagement et de la fatigue des métaux ductiles. Ph.D. Thesis, spécialité. Mécanique, Université de Lille I.

Orowan, E., 1939. Theory of the fatigue of metals. Proc. Royal Soc. 171, 79-106.

Papadopoulos, I.V., 1987. Fatigue polycyclique des métaux: une nouvelle approche. Ph.D. Thesis, spécialité. Mécanique, Ecole des Ponts et Chaussées, France. Papadopoulos, I.V., 1996. Exploring the high-cycle fatigue behaviour of metals from the mesoscopic scale. J. Mech. Behav. Mater. 6 (2), $93-118$.

Rice, J.R., Tracey, D.M., 1969. On a ductile enlargement of voids in triaxial stress fields. J. Mech. Phys. Solids 17, 201-217.

Ros, M., 1950. Die bruchegefahr fester köper. Technical report, EMPA Bericht 173, Zurich.

Sines, G., 1959. Metal Fatigue. McGraw Hill.

Suresh, S., 1998. Fatigue of Materials, second ed. Cambridge University Press, Cambridge.

Winter, A.T., 1974. A model for the fatigue of copper at low plastic strain amplitude. Philos. Mag. 30 (4), 719-738. 